Research Paper

\title{
Implications of Type1/2 Diabetes Mellitus in Breast Cancer Development: A General Female Population-based Cohort Study
}

\author{
Yung-Po Liaw ${ }^{1,2}$, Pei-Chieh Ko1, Shiou-Rung Jan¹, Jing-Yang Huang1, Oswald Ndi Nfor' ${ }^{1}$, Chia-Chi Lung1,2, \\ Yi-Chen Chiang, ${ }^{1,2}$, Liang-Tsai Yeh³, Ming-Chih Chou ${ }^{3}$, Horng-Der Tsai4, Yi-Hsuan Hsiao ${ }^{3,4}, \bowtie$ \\ 1. Department of Public Health and Institute of Public Health, Chung Shan Medical University, Taichung, Taiwan \\ 2. Department of Family and Community Medicine, Chung Shan Medical University Hospial, Taichung, Taiwan \\ 3. School of Medicine, Chung Shan Medical University, Taichung, Taiwan \\ 4. Department of Obstetrics and Gynecology, Changhua Christian Hospital, Changhua, Taiwan
}

$\square$ Corresponding author: Yi-Hsuan Hsiao, M.D., PhD, School of Medicine, Chung Shan Medical University, Department of Obstetrics and Gynecology, Changhua Christian Hospital, 135 Nanhsiao Street, Changhua, 500 Taiwan. Phone: +886-4-7238595; Fax: +886-4-7228289; E-mail: 54315@cch.org.tw

() 2015 Ivyspring International Publisher. Reproduction is permitted for personal, noncommercial use, provided that the article is in whole, unmodified, and properly cited. See http:/ /ivyspring.com/terms for terms and conditions.

Received: 2015.03.21; Accepted: 2015.05.25; Published: 2015.06.25

\begin{abstract}
Aim: The current study assessed the potential impact of diabetes type 1 and type 2 for female breast cancer risk.

Materials and Methods: The health information and medical record of the entire adult female residents in Taiwan were retrieved from Taiwan's National Health Insurance Research Database. Multivariate Cox proportional hazard regression models and descriptive statistics were used to identify potential correlations between type $1 / 2$ diabetes and breast cancer. In addition, this study statistically assessed the possible association of diabetes and breast cancer risk with age, insurance amount (quality of care), and regions.

Results: The diabetic status of the entire adult female population was assessed between 2001 and 2003. Of $10,827,079$ adult females, $4,738(0.04 \%)$ were diagnosed with type 1 and $830,546(7.7 \%)$ with type 2 diabetes, and 9, 991,795 (92.3\%) were free of diabetes. From 2004 to 2010, a total of 57,283 cases of breast cancer were detected, with an average breast cancer incidence rate of $0.53 \%$ in the generation population. The actual breast cancer incidence rate was $0.30 \%$ ( 14 of $4,738)$ in patients with type 1 diabetes, $1.10 \%(9,105$ of 830,546$)$ in patients with type 2 diabetes, and $0.48 \%(48,164$ of $9,991,795)$ in patients free of diabetes. The breast cancer incidence rate is significantly higher $(p<0.001)$ in patients with type 2 diabetes than that in patients with type 1 diabetes and in patients free of diabetes.

After adjusting for the covariates of age, insurance cost, and region, hazard ratios (HRs) for the association between breast cancer risk and types 1 and $2 \mathrm{DM}$ were $1.01(\mathrm{Cl}=0.60-1.71)$ and 1.13 $(\mathrm{Cl}=1.10-1.16)$, respectively. Women with type 2 diabetes were at a significantly higher risk for development of breast cancer compared with those free of diabetes, but there appeared to have no significant increase in risk for those with type 1 diabetes. Our study also revealed that age, insurance amount (quality of care), and region are significantly associated with diabetes and breast cancer risk $(\mathrm{p}<0.0001)$.

Conclusion: Our results demonstrated different implications of diabetes type for the risk of breast cancer with type 2 posing a higher risk than type 1. This is the largest cohort study that assesses the possible correlation between both type 1 and 2 diabetes with breast cancer, and also is the largest cohort study showing that diabetes are associated with age, insurance, and region,
\end{abstract}


which further suggest that living condition and life style may significantly associated with diabetes and breast cancer.

Key words: diabetes, breast cancer, cohort studies, Cox model

\section{Introduction}

Diabetes is a serious public health concern worldwide with a predicted increase in global prevalence of $69 \%$ and $20 \%$ in developing and developed countries, respectively, between 2010 and 2030, thereby depicting a growing burden of this disease (1). In addition, breast cancer remains a significant health liability worldwide with the global burden reported to have doubled between 1975 and 2000, with an additional two-fold increase expected before 2030 (2). In Taiwan, the incidence of breast cancer (the most common female cancer since 2003) has increased during the past 2 decades (3). Although medical expenses associated with breast cancer treatments have significantly increased, the incidence and mortality rate have not decreased (4).

Although breast cancer and diabetes are associated with different etiologies, both are influenced by several factors, including genetic and environmental, and a relationship between these diseases has been established (5). However, a previous study showed that the correlation between the incidence of diabetes and breast cancer was only significant among populations in Europe [relative risk $(\mathrm{RR})=1.88$; 95\% confidence interval $(\mathrm{CI})=1.56-2.25]$ and the United States $(\mathrm{RR}=1.16 ; 95 \% \mathrm{CI}=1.12-1.20)$ but not Asia $(\mathrm{RR}=$ $1.01 ; 95 \% \mathrm{CI}=0.84-1.21)$ (6). Diabetes type 1 is generally not considered as a risk factor of breast cancer because type 1 diabetic patients are younger and the risk of breast cancer increases by age $(7,8)$; an increasing incidence of breast cancer in young women has been reported (9-13). A retrospective cohort study conducted in China reported that patients with type 2 diabetes mellitus (DM) were at an increased risk of developing breast cancer $(\mathrm{RR}=2.21 ; 95 \% \mathrm{CI}=$ 1.49-2.93) (14). However, previous studies did not investigate the association between breast cancer and type 1 vs. type $2 \mathrm{DM}$ in the same population. Therefore, our current study attempted to demonstrate association between types 1/ $2 \mathrm{DM}$ and breast cancer risk in Taiwan.

\section{Methods}

In Taiwan, a cancer registry was started in 1979 and the National Health Insurance (NHI) service was implemented in 1995. The NHI covers comprehensive medical care, including preventative health care, as well as outpatient and inpatient services. Taiwan's National Health Insurance Research Database
(TNHIRD) is derived from the NHI database and made available to scientists nationwide for use in research. We retrieved data for this study from TNHIRD, which contains detailed clinical records of every patient during each visit, primary and secondary diagnostic codes, as well as prescription orders. Diagnostic guidelines from the International Classification of Diseases, Ninth Revision, Clinical Modification (ICD-9-CM) were used to identify the diseases of interest.

Identifying information in TNHIRD is encrypted before release and each patient has a fixed transcode. The fixed transcode facilitated evaluation of the same patient population in this study.

Ambulatory care expenditures by visit (CD) or inpatient expenditures by admission (DD) incurred by patients with DM from 2001 to 2003 were identified from TNHIRD. Individuals with breast cancer were identified from the 2004-2010 databases. The main steps of the study were elucidated in figure 1.

Demographic characteristics, such as insurance cost and geographical location, were adjusted for confounding factors. Insurance cost was categorized into four levels based on personal monthly income: family dependent; < New Taiwan dollar (NT\$) 20,000; NT\$20,000-NT\$40,000; and >NT\$40,000 (1.0 USD is about 31-34 Taiwan Dollars). The six main categories of the NHI were based on geographic and administrative districts: (i) Taipei, (ii) Northern, (iii) Central, (iv) Southern, (v) Kao-Ping, and (vi) Eastern region.

All statistical analyses were performed using the SAS statistical package v.9.2 (SAS Institute Inc., Cary, NC, USA). Analysis of variance was used to analyze differences in age among patients with types 1 and 2 $\mathrm{DM}$ and non-DM subjects as controls. The chi-square test was used to identify associations among categorical data. In addition, we used multivariate Cox proportional hazard regression models to identify associations between DM (independent variable) and breast cancer risk (dependent variable). Insurance cost and geographic characteristics were adjusted for confounding factors. A probability $(P)$ value of $<0.05$ was considered statistically significant.

\section{Results}

A flow chart illustrating the selection of cases for this study is shown in Figure 1. Characteristics of women with and without diabetes obtained from 
medical records from 2001 to 2003 are shown in Table 1 . The diabetic status of the entire adult female population was assessed between 2001 and 2003. Of $10,827,079$ adult females, 4,738 (0.04\%) were diagnosed with type 1 and 830,546 (7.7\%) with type 2 diabetes, and 9, 991,795 (92.3\%) were free of diabetes (Figure 1, Table 1). From 2004 to 2010, a total of 57,283 cases of breast cancer were detected, with an average breast cancer incidence rate of $0.53 \%$ in the generation population. The actual breast cancer incidence rate was $0.30 \%(14$ of 4,738$)$ in patients with type 1 diabetes, $1.10 \%(9,105$ of 830,546$)$ in patients with type 2 diabetes, and $0.48 \%(48,164$ of $9,991,795)$ in patients free of diabetes (Table 2, Table 3). The breast cancer incidence rate is significantly higher $(p<0.001)$ in patients with type 2 diabetes than that in patients with type 1 diabetes and in patients free of diabetes (Table $3)$.

There were significant differences in patient age, insurance cost, region, and number of breast cancer cases among the type $1 \mathrm{DM}$, type $2 \mathrm{DM}$, and non-DM groups. The mean $( \pm S D)$ ages of women in the type 1
$\mathrm{DM}$, type $2 \mathrm{DM}$, and non-DM groups were 24.67 $( \pm 14.62), 60.16( \pm 13.91)$, and $33.06( \pm 19.05)$ years, respectively (Table 1). Our study also revealed that age, insurance amount (quality of care), and region are significantly associated with diabetes and breast cancer risk $(\mathrm{p}<0.0001)$.

Cox regression analysis of breast cancer development according to DM, age, insurance cost (dependent, <NT\$20,000, NT\$20,000-39,000, and $>$ NT\$40,000), and region (Taipei, Northern, Central, Southern, Kao-Ping, and Eastern) was performed. After adjusting for the covariates of age, insurance cost, and region, hazard ratios (HRs) for the association between breast cancer risk and types 1 and $2 \mathrm{DM}$ were $1.01(\mathrm{CI}=0.60-1.71$, Table 4$)$ and $1.13(\mathrm{CI}=$ 1.10-1.16, Table 5), respectively. Taken together, our results indicate that women with type $2 \mathrm{DM}$ were at a significantly higher risk for development of breast cancer compared with those in the non-DM group, but there was no significant increase in risk for those with type $1 \mathrm{DM}$.

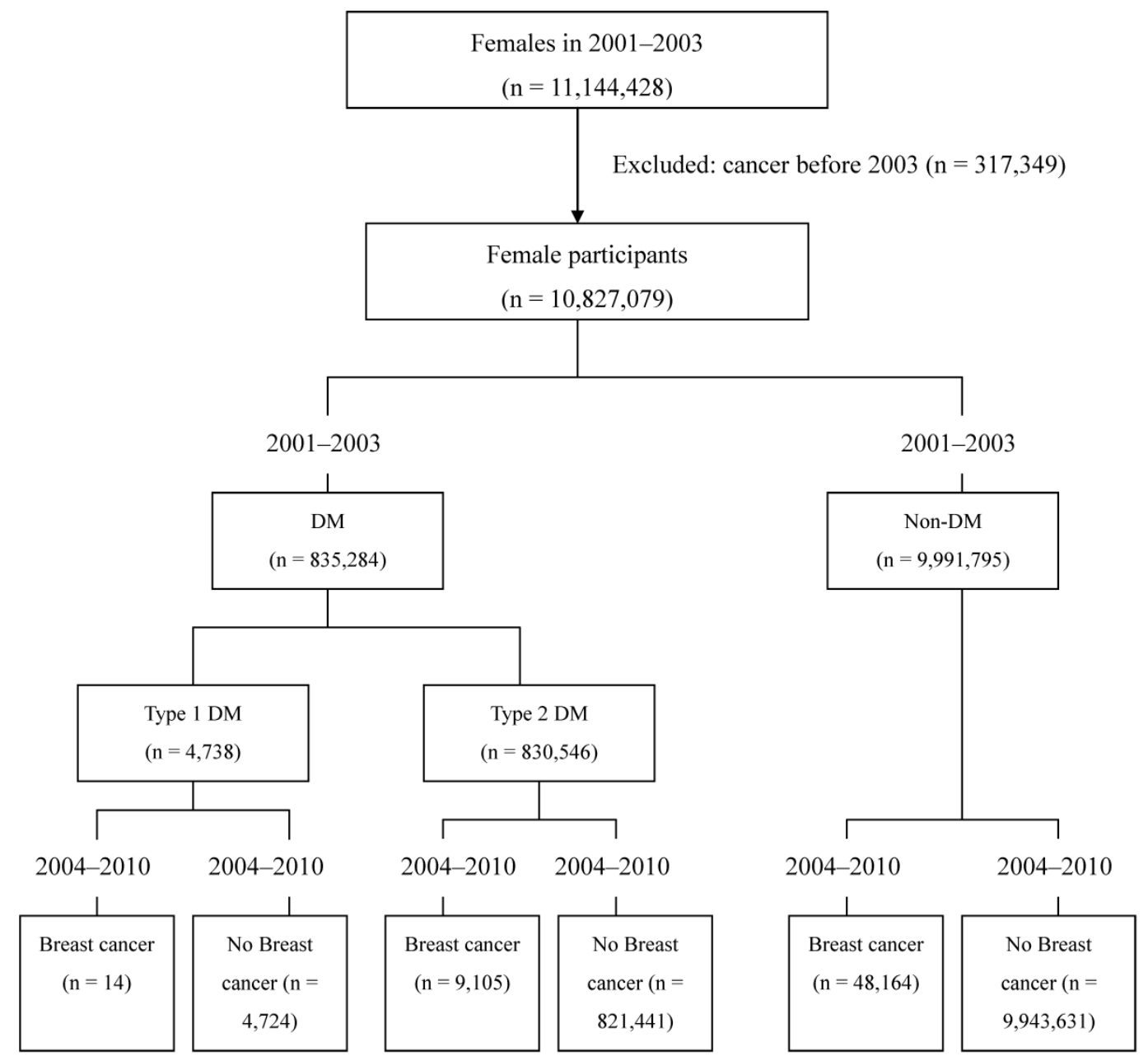

Figure 1. Flow chart of this study 
Table 1. Characteristics of diabetic and nondiabetic women from 2001 to 2003

\begin{tabular}{|c|c|c|c|c|c|c|c|}
\hline Variable & $\begin{array}{l}\text { Type 1 DM } \\
(\mathrm{n}=4738)\end{array}$ & & $\begin{array}{l}\text { Type } 2 \text { DM } \\
(\mathrm{n}=830546)\end{array}$ & & $\begin{array}{l}\text { Non-DM } \\
(\mathrm{n}=9991795)\end{array}$ & & $P$ value \\
\hline & No. & $\%$ & No. & $\%$ & No. & $\%$ & \\
\hline Age (y) & $24.67 \pm 14.62$ & & $60.16 \pm 13.91$ & & $33.06 \pm 19.05$ & & $<0.0001$ \\
\hline $\begin{array}{l}\text { Insurance amount *Taiwan } \\
\text { Dollars }\end{array}$ & & & & & & & $<0.0001$ \\
\hline Dependent & 2667 & 56.29 & 300393 & 36.17 & 4543005 & 45.47 & \\
\hline$<20,000$ & 1313 & 27.71 & 391687 & 47.16 & 3305147 & 33.08 & \\
\hline $20,000-39,999$ & 578 & 12.2 & 107592 & 12.95 & 1509668 & 15.11 & \\
\hline$\geq 40,000$ & 180 & 3.8 & 30874 & 3.72 & 633975 & 6.34 & \\
\hline Region & & & & & & & $<0.0001$ \\
\hline Taipei & 1754 & 37.02 & 276926 & 33.34 & 3676116 & 36.79 & \\
\hline Northern & 660 & 13.93 & 104078 & 12.53 & 1433682 & 14.35 & \\
\hline Central & 936 & 19.76 & 149436 & 17.99 & 1841211 & 18.43 & \\
\hline Southern & 570 & 12.03 & 136627 & 16.45 & 1343467 & 13.45 & \\
\hline Kao-Ping & 656 & 13.85 & 138827 & 16.72 & 1473991 & 14.75 & \\
\hline Eastern & 162 & 3.42 & 24652 & 2.97 & 223328 & 2.24 & \\
\hline $\begin{array}{l}\text { Breast cancer (developed in } \\
2004-2010 \text { ) }\end{array}$ & & & & & & & $<0.0001$ \\
\hline Yes & 14 & 0.30 & 9105 & 1.10 & 48164 & 0.48 & \\
\hline Non & 4724 & 99.70 & 821441 & 98.90 & 9943631 & 99.52 & \\
\hline
\end{tabular}

Age was mean \pm s.d.

DM, diabetes mellitus

*1.0 USD is about 31-34 Taiwan Dollars

Table 2. Comparison of breast cancer incidences among different populations

\begin{tabular}{lllll}
\hline Category & Total case number & $\begin{array}{l}\text { Total cancer } \\
\text { cases }\end{array}$ & $\begin{array}{l}\% \text { of } \\
\text { cancer }\end{array}$ & $\mathrm{p}$ \\
\hline Generation population & $10,827,079$ & 57,283 & $0.53 \%$ & \\
Non-diabetic population & $9,991,795$ & 48,164 & $0.48 \%$ & $<0.001$ \\
Diabetic population & 835,284 & 9,119 & $1.09 \%$ & \\
\hline
\end{tabular}

Table 3. Comparison of breast cancer incidences between diabetic and non-diabetic populations

\begin{tabular}{lllll}
\hline Category & $\begin{array}{l}\text { Total case } \\
\text { number }\end{array}$ & $\begin{array}{l}\text { Total cancer } \\
\text { cases }\end{array}$ & $\begin{array}{l}\% \text { of } \\
\text { cancer }\end{array}$ & $\mathrm{p}$ \\
\hline Non-diabetic population & $9,991,795$ & 48,164 & $0.48 \%$ & \\
Diabetic population & 835,284 & 9,119 & $1.09 \%$ & $<0.001$ \\
Type 1 & 4,738 & 14 & $0.30 \%$ & \\
Type 2 & 830,546 & 9,105 & $1.10 \%$ & \\
\hline
\end{tabular}

Table 4. Adjusted hazard ratios (HRs) and $95 \%$ confidence interval $(\mathrm{Cl})$ for the association between type $1 \mathrm{DM}$ and breast cancer risk

\begin{tabular}{lll}
\hline Variable & \multicolumn{2}{l}{ Cox regression model } \\
\cline { 2 - 3 } & HR & $95 \% \mathrm{CI}$ \\
\hline Type1 DM & ref & - \\
Non & 1.01 & $0.60-1.71$ \\
Yes & 1.04 & $1.042-1.043$ \\
Age & & \\
Insurance amount (Taiwan Dol- & & \\
lars) & 0.69 & $0.67-0.70$ \\
Dependent & ref & - \\
l<20,000 & 1.40 & $1.36-1.43$ \\
$20,000-39,999$ & 2.02 & $1.97-2.08$ \\
$\geq 40,000$ & & \\
Region & ref & - \\
Taipei & 0.81 & $0.78-0.83$ \\
Northern & 0.84 & $0.82-0.87$ \\
Central & 0.78 & $0.76-0.80$ \\
Southern & 0.90 & $0.88-0.93$ \\
Kao-Ping & 0.78 & $0.73-0.83$ \\
Eastern & & \\
\hline
\end{tabular}

DM, diabetes mellitus
Table 5. Adjusted hazard ratios (HRs) and $95 \%$ confidence interval $(\mathrm{Cl})$ for the association between type $2 \mathrm{DM}$ and breast cancer risk

\begin{tabular}{lll}
\hline Variable & \multicolumn{2}{l}{ Cox regression model } \\
\cline { 2 - 3 } & HR & $95 \%$ CI \\
\hline Type2 DM & ref & - \\
Non & 1.13 & $1.10-1.16$ \\
Yes & 1.04 & $1.039-1.04$ \\
Age & & \\
Insurance amount (Taiwan Dol- & & \\
lars) & & \\
Dependent & 0.73 & $0.71-0.75$ \\
1<20,000 & ref & - \\
$20,000-39,999$ & 1.42 & $1.39-1.46$ \\
$\geq 40,000$ & 2.03 & $1.97-2.08$ \\
Region & & \\
Taipei & ref & - \\
Northern & 0.80 & $0.78-0.82$ \\
Central & 0.84 & $0.82-0.86$ \\
Southern & 0.77 & $0.75-0.79$ \\
Kao-Ping & 0.89 & $0.87-0.91$ \\
Eastern & 0.78 & $0.74-0.83$ \\
\hline
\end{tabular}

DM, diabetes mellitus

\section{Discussion}

Most studies for the identification of risk factors for breast cancer among women with diabetes have concentrated on type $2 \mathrm{DM}$ and have failed to discriminate between types 1 and 2 . Therefore, any correlations to type $1 \mathrm{DM}$ remain dubious (15). We conducted a general female population-based cohort study to assess the implications of type $1 / 2$ diabetes mellitus in breast cancer development. A previous meta-analysis reported that the correlation between diabetes and breast cancer was not significant among the Asian population $(\mathrm{RR}=1.01 ; 95 \% \mathrm{CI}=0.84-1.21)$, although a significant association was found in stud- 
ies conducted in Europe $(\mathrm{RR}=1.88 ; 95 \% \mathrm{CI}=$ 1.56-2.25) and the United States $(\mathrm{RR}=1.16 ; 95 \% \mathrm{CI}=$ 1.12-1.20) (6). However, in these studies, the authors did not clarify the type of diabetes. Furthermore, a meta-analysis conducted by Hardefeldt et al. (16) confirmed an association between type $2 \mathrm{DM}$ and breast cancer, but an insufficient number of studies were pooled to calculate the odds ratio of breast cancer risk associated with type $1 \mathrm{DM}$.

To the best of our knowledge, the present population-based study is the first to differentiate the correlations of types 1 and $2 \mathrm{DM}$ to the risk of breast cancer development. Our results clearly indicated a significantly increased risk of breast cancer among women with type $2 \mathrm{DM}$ but not among those with type 1. Because case-control studies are susceptible to recall, and selection biases and the possibility of publication bias is inherent in meta-analyses of published studies, we used a population-based cohort study to identify relationships between the risk of breast cancer among women with type 1 or 2 DM.

The results of our study were consistent with those of a population-based cohort study conducted in Sweden (17), which found no statistically significant increase in the risk of breast cancer among women with type $1 \mathrm{DM}$ compared with those women without diabetes; however, the incidence among women with type 2 DM was not addressed in that study.

Types 1 and 2 DM differ in metabolic characteristics and hormonal status. Hyperglycemia is associated with deficient endogenous insulin secretion in type 1 , whereas hyperglycemia and hyperinsulinemia coexist in type 2 because of insulin resistance in peripheral tissues. Type $1 \mathrm{DM}$ is an autoimmune disease with no known prevention, where type $2 \mathrm{DM}$ is usually associated with older age, obesity, and physical inactivity. Thus type $2 \mathrm{DM}$ is preventable through maintenance of healthy body weight, healthy food choices, and physical activity. Type $1 \mathrm{DM}$ accounts for approximately $5 \%$ of diabetes cases and is usually diagnosed in children and young adults. The aim of our study was to interpret epidemiological evidence between the association of diabetes and risk of breast cancer. In this population-based cohort study, a significant difference was identified in the risk of breast cancer among patients with type 1 and 2 DM. However, because type $1 \mathrm{DM}$ occurs less frequently, we were concerned that the low number of such patients may have biased our results. The different implications of types 1 and $2 \mathrm{DM}$ in breast cancer may be associated with the biological characteristics of each subset.

Several biological mechanisms for the association between diabetes and breast cancer have been proposed, including hyperinsulinemia, hyperglycemia, and inflammation; however, the results of studies to identify potential biological links between diabetes and breast cancer were inconclusive $(15,18)$. Patients with type $2 \mathrm{DM}$ develop chronic hyperglycemia and hyperinsulinemia due to insulin resistance in peripheral tissues. The role of insulin in promoting cancer growth was first shown in an in vivo study of insulin administration to intact, oophorectomized, and hypophysectomized rats to demonstrate intrinsic growth-stimulating effects of insulin on rat mammary tumor tissue; then insulin-dependent mammary carcinoma formation was detected (19). Hyperinsulinemia is suspected of promoting cell proliferation pathways in carcinogenesis through activation of insulin receptors (18); therefore, hyperinsulinemia in association with insulin resistance may be a significant risk factor in the development of breast cancer, independent of body fat distribution or general obesity (20). In addition, increased leptin production and decreased adiponection production by adipose tissue may contribute to the development and progression of breast cancer among obese patients and those with type 2 DM (21).

The risk of mortality from breast cancer is higher among women with diabetes than those without diabetes $(5,22,23)$. The risk of breast cancer among women with diabetes, particularly type 2 , may be a clinical and economic burden in the future because of the increasing incidence of diabetes worldwide. Therefore, further investigations are required to develop regimens to better coordinate treatments for diabetes and breast cancer, as well as methods for both disease screening and prevention.

However, our study had some limitations. First, there may have been confounding factors impacting the association of diabetes and breast cancer risk because these diseases share several risk factors. Residual confounding factors could not be completely excluded though the risk estimates that were adjusted for possible confounding factors in most epidemiological studies (15). Several known risk factors of breast carcinogenesis were not controlled in the multivariate analysis, for instance, nulliparity/multiparity, use of hormone supplementation or hormonal contraception, smoking history, family history of breast cancer, and alcohol consumption. Data retrieved from TNHIRD did not include this information. Patients with type $1 \mathrm{DM}$ are usually younger (Table 1); thus, it was impractical to include nulliparity/multiparity, use of hormones, smoking history, or alcohol consumption in the multivariate analysis. In our study, we adjusted for the demographic characteristics of age, insurance cost, and geographical region. Second, the analysis for type 1 
diabetes and risk of breast cancer may be underpowered because type 1 diabetes is diagnosed in young individuals and there is not enough follow-up on those individuals who may develop breast cancer. Lastly, women included in the non-DM group may develop type $2 \mathrm{DM}$ beyond the years we looked at; therefore, the HR values may have been underestimated in this study.

Our results demonstrated different implications of diabetes type for the risk of breast cancer with type 2 posing a higher risk than type 1 . This is the largest cohort study that assesses the possible correlation between both type 1 and 2 diabetes with breast cancer, and also is the largest cohort study showing that diabetes are associated with age, insurance, and region, which further suggest that living condition and life style may significantly associated with diabetes and breast cancer.

\section{Acknowledgements}

This study is based in part on data from the National Health Insurance Research Database provided by the Bureau of National Health Insurance, Department of Health and managed by National Health Research Institutes. The interpretation and conclusions contained herein do not represent those of the Bureau of National Health Insurance, the Department of Health or National Health Research Institutes. The authors appreciate Dr. Susan Olmstead-Wang's English editing of this paper.

\section{Competing interests}

The authors declare that they have no competing interests.

\section{References}

1. Shaw JE, Sicree RA, Zimmet PZ. Global estimates of the prevalence of diabetes for 2010 and 2030. Diabetes Res Clin Pract. 2010; 87: 4-14.

2. Boyle P, Howell A. The globalisation of breast cancer. Breast Cancer Res. 2010; 12 (Suppl 4): S7.

3. Chang KJ, Kuo WH, Wang MY. The epidemiology of breast cancer in Taiwan. J Chin Oncol. 2008; 24: 85-93.

4. Ho ML, Liaw YP, Lai CH, Chen YY, Tsai HD, Chou MC, Hsiao YH. Significantly increased medical expenditure on breast cancer failing to bring down its mortality and incidence rate. J Cancer. 2013; 4: 531-5.

5. Cleveland RJ, North KE, Stevens J, Teitelbaum SL, Neugut AI, Gammon MD. The association of diabetes with breast cancer incidence and mortality in the Long Island Breast Cancer Study Project. Cancer Causes Control. 2012; 23: 1193-203.

6. Liao S, Li J, Wei W, Wang L, Zhang Y, Li J, Wang C, Sun S. Association between diabetes mellitus and breast cancer risk: a meta-analysis of the literature. Asian Pac J Cancer Prev. 2011; 12: 1061-5.

7. Desantis C, Ma J, Bryan L, Jemal A. Breast cancer statistics. CA Cancer J Clin. 2013; 64: 52-62.

8. Siegel R, Naishadham D, Jemal A. Cancer statistics,2012. CA Cancer J Clin. 2012; 62: 10-29.

9. Arleo EK, Reichman M, Dashevsky BZ, Babagbemi K, Drotman M. Breast cancer in women in their thirties (2007-2013): A retrospective review. Breast Dis. 2015; [Epub ahead of print]

10. Son BH, Dominici LS, Aydogan F, et al. Young women with breast cancer in the United States and South Korea: comparison of demographics, pathology and management. Asian Pac J Cancer Prev. 2015; 16: 2531-5.

11. Reyna C, Lee MC. Breast cancer in young women: special considerations in multidisciplinary care. J Multidiscip Healthc. 2014; 7: 419-29.
12. Gewefel $\mathrm{H}$, Salhia B. Breast cancer in adolescent and young adult women. Clin Breast Cancer. 2014; 14: 390-5.

13. Partridge $\mathrm{AH}$, Pagani $\mathrm{O}$, Abulkhair $\mathrm{O}$, et al. First international consensus guidelines for breast cancer in young women (BCY1). Breast. 2014; 23: 209-20.

14. Zhang PH, Chen $\mathrm{ZW}, \mathrm{Lv} \mathrm{D}$, et al. Increased risk of cancer in patients with type 2 diabetes mellitus: a retrospective cohort study in China. BMC Public Health. 2012; 12: 567.

15. Shikata $K$, Ninomiya $T$, Kiyohara $Y$. Diabetes mellitus and cancer risk: review of the epidemiological evidence. Cancer Sci. 2013; 104: 9-14.

16. Hardefeldt PJ, Edirimanne S, Eslick GD. Diabetes increases the risk of breast cancer: a meta-analysis. Endocr Relat Cancer. 2012; 19: 793-803.

17. Zendehdel K, Nyren O, Ostenson CG, Adami HO, Ekbom A, Ye W. Cancer incidence in patients with type 1 diabetes mellitus: a population-based cohort study in Sweden. J Natl Cancer Inst. 2003; 95: 1797-800.

18. Giovannucci E, Harlan DM, Archer MC, et al. Diabetes and cancer: a consensus report. Diabetes Care. 2010; 33: 1674-85.

19. Heuson JC, Legros N, Heimann R. Influence of insulin administration on growth of the 7,12-dimethylbenz(a)anthracene-induced mammary carcinoma in intact, oophorectomized, and hypophysectomized rats. Cancer Res. 1972; 32: $233-8$.

20. Bruning PF, Bonfrer JM, van Noord PA, Hart AA, de Jong-Bakker M, Nooijen WJ. Insulin resistance and breast-cancer risk. Int J Cancer. 1992; 52: 511-6.

21. Vona-Davis L, Howard-McNatt M, Rose DP. Adiposity, type 2 diabetes and the metabolic syndrome in breast cancer. Obes Rev. 2007; 8: 395-408.

22. Yancik R, Wesley MN, Ries LA, Havlik RJ, Edwards BK, Yates JW. Effect of age and comorbidity in postmenopausal breast cancer patients aged 55 years and older. JAMA. 2001; 285: 885-92.

23. Tseng $\mathrm{CH}$, Chong CK, Tai TY. Secular trend for mortality from breast cancer and the association between diabetes and breast cancer in Taiwan between 1995 and 2006. Diabetologia. 2009; 52: 240-6. 